\title{
Hyperlipidemia in post-COVID patients; a unique observational follow-up study on lipid levels in post-COVID patients
}

\author{
๑D Ŏuz Abdullah Uyaroğlu, @Murat Özdede, @Nursel Çalık Başaran, ĐBahadır Köylü, \\ ๑Taha Koray Şahin, @Lale Özışık, @Mine Durusu Tanrıöver, @Gülay Sain Güven \\ Hacettepe University, Faculty of Medicine, Department of Internal Medicine, Section of General Internal Medicine, Ankara, Turkey
}

Cite this article as: Uyaroğlu OA, Özdede M, Çalık Başaran N, et al. Hyperlipidemia in Post-COVID patients; a unique observational followup study on lipid levels in post-COVID patients. J Health Sci Med 2022; 5(1): 220-226.

\begin{abstract}
Aim: Alterations in plasma lipid levels have been shown to be correlated with the severity of infections due to various pathogens such as bacteria, viruses. In this study, we aimed to evaluate the lipid metabolism changes associated with disease severity and prognosis in hospitalized COVID-19 patients during and after (post-COVID) the disease.

Material and Method: Patients who were hospitalized in the COVID-19 wards between April 02, 2020, and November 20, 2020 and were then evaluated in the follow-up outpatient clinic were retrospectively searched.

Results: Lipid levels were present at the admission and follow-up for 95 patients. The mean (S.D) age was 48.49 (16.4), and 49(51.6\%) were male. The mean (S.D) day between the admission and the first visit in the COVID-19 follow-up outpatient clinic was 27.8 (12.8). LDL-C ( $\mathrm{p}=0.044)$, and HDL-C $(\mathrm{p}=0.004)$ levels were significantly lower in the severely ill group at the admission. Total cholesterol, LDL-C, HDL-C, and triglyceride levels on follow-up were significantly higher than those levels on the admission day $(\mathrm{p}<0.001)$. Delta (Follow up-Admission) levels LDL-C, total cholesterol and triglyceride levels were significantly high in patients who have received steroid therapy. Only delta LDL-C was significantly high in patients who require Intensive Care Unit.

Conclusions: Dyslipidemia is observed in COVID-19 patients both during the disease and in the post-COVID period. Our findings also support the evidence demonstrating that low LDL-C and/or HDL-C levels can increase the risk of developing severe infections, also in COVID-19. The dynamics of lipid profiles before/during and after the entire disease course should be monitorized.
\end{abstract}

Keywords: COVID-19, dyslipidemia, hyperlipidemia, post-COVID, long-COVID

\section{INTRODUCTION}

Although almost two years have been since the start of the Coronavirus Disease 2019 (COVID-19) pandemic, data on the pathophysiology, predictors of severity, and treatment of the disease are still incomplete. To date, thousands of reports have emerged on almost every aspect of COVID-19 and the literature is still growing with new pieces of evidence. However, we still have limited information regarding lipoproteins and COVID-19.

Acute infections have been shown to lead to significant alterations in metabolic regulation, including lipids and lipoproteins, which play a central role in the host immune response (1). The common lipid alterations include a decrease in total cholesterol (TC) and an increase in the concentration of triglyceride (TG) -rich lipoproteins. Additionally, low- and highdensity lipoprotein - cholesterol (LDL-C and HDL-C, respectively), apolipoprotein-A1, and apolipoprotein-B levels decrease (1-3). The role of lipid levels as a marker of infection severity and prognosis has been investigated and HDL-C, apo-A1 and LDL-C have been shown as prognostic markers in patients with sepsis, pneumonia and other infections $(4,5)$.

Alterations in lipid and lipoproteins in COVID-19 patients have been observed and dyslipidemia has been associated with the inflammatory response, disease severity and poor prognosis. LDL-C, TC and HDL-C concentrations significantly decreased in COVID-19 patients (6-10). Although most of the studies were in 
hospitalized patients, literature on lipid profiles during follow-up in discharged COVID-19 (post-COVID) patients is rare (11-12).

In this real life, observational follow-up study, we aimed to evaluate the association of lipid metabolism changes with disease severity and prognosis in hospitalized COVID-19 patients during and after the disease.

\section{MATERIAL AND METHOD}

The study was carried out with the permission of Hacettepe University Non-Interventional Clinical Researchs Ethics Committee (Date: 31.03.2020, Decision No: 20/353). All procedures were carried out in accordance with the ethical rules and the principles of the Declaration of Helsinki.

\section{Study Design and Population}

This study was conducted in an accredited tertiary care hospital. A "COVID-19 follow-up outpatient clinic" was established in the initial stages of the pandemic to address and manage the clinical needs of non-critical COVID-19 adult patients after discharge (post-COVID). Patients who were hospitalized in the COVID-19 wards were scheduled a follow-up visit in the 2-4 weeks at the time of discharge.

Patients who were hospitalized in the COVID-19 wards between April 02, 2020, and November 20, 2020 and were then evaluated in the follow-up outpatient clinic were retrospectively searched from a prospectively formed database. Patients who had polymerase chain reaction (PCR) confirmed COVID-19 were screened. Those who had plasma lipid levels measured at the time of admission in the COVID-19 wards and in the COVID-19 follow-up outpatient clinic thereafter were included. As this was a retrospective study, there was no intervention to the management of the patients. Patients on statin treatment continued to take their drug while those who were not on statins were not initiated statin treatment during the study period.

The clinical severity of the patients was graded as mild, moderate or severe according to the World Health Organization (WHO) classification (13). Patients who were graded as critical at the time of admission and hospitalized directly to intensive care units (ICU) were not included.

\section{Statistical Analysis}

Statistical analysis was performed with IBM SPSS for Windows version 23 package. Normally distributed continuous data were summarized by mean \pm standard deviation (SD), while non-normally distributed continuous data were summarized by median $\left[25-75^{\text {th }}\right.$ percentiles]. The Chi-square test or Fisher exact test were applied to detect the relation between categorical variables. Independent sample t-test or Mann Whitney U test was used to compare independent two groups in terms of numerical data. Within group differences were shown by Wilcoxon test. A 2-tailed $p$ value of 0.05 was considered significant.

\section{RESULTS}

A total of 1105 adult patients with laboratory confirmed COVID-19 were hospitalized in COVID-19 wards. Plasma lipid levels of 108 patients were available at the time of admission, among whom 95 had been evaluated in the follow-up clinic and plasma lipid levels were available (Figure 1).

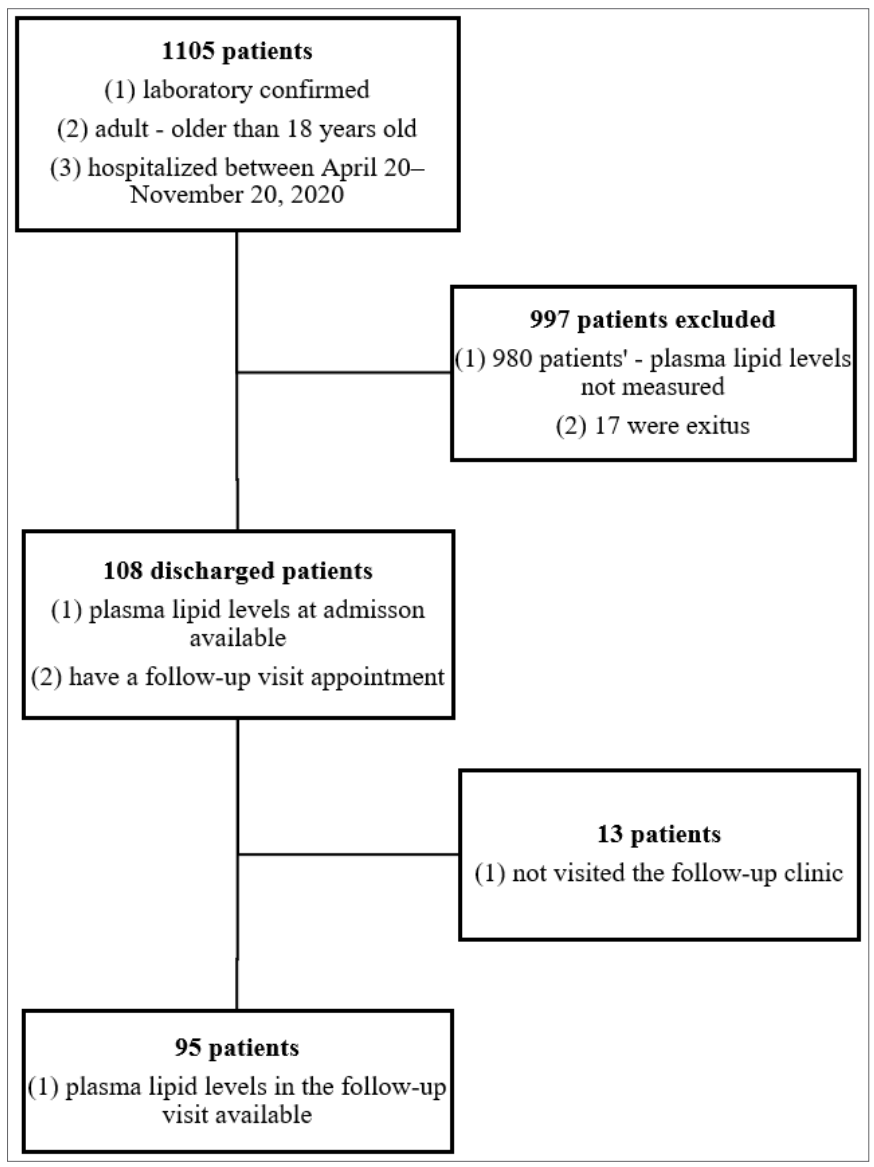

Figure 1. Flowchart of the patient selection

\section{Baseline Characteristics and Plasm Lipid Values at the Time of Admission}

The mean (SD) age of the patients was 48.5 (16.4) years, and $49(51.6 \%)$ were male. The median length of stay $(\mathrm{LoS})$ was $6(\mathrm{IQR}=5)$ days. Baseline characteristics of patients and plasma lipid levels at admission are given in Table 1.

The median time from symptom onset to hospital admission (on the day of venous sampling for lipid parameters) was $3(\mathrm{IQR}=4)$ days. The median (IQR) HDL-C, LDL-C, TC and TG levels were 38.1 (15.1), 106.8 (52), 168.2 (62.1) and 119.5 (89) mg/dL, respectively. 


\begin{tabular}{|c|c|c|c|c|c|c|c|c|c|}
\hline & $\begin{array}{c}\text { Total, } \\
\text { n=95 (\%) }\end{array}$ & $\begin{array}{c}\text { HDL-C } \\
\text { median } \\
\text { (IQR), } \\
\text { mg/dL }\end{array}$ & $\mathbf{P}$ & $\begin{array}{c}\text { LDL-C } \\
\text { median } \\
\text { (IQR), } \\
\text { mg/dL }\end{array}$ & $\mathbf{P}$ & $\begin{array}{c}\text { Triglycerides } \\
\text { median } \\
\text { (IQR), } \\
\text { mg/dL }\end{array}$ & $\mathbf{P}$ & $\begin{array}{c}\text { Total } \\
\text { cholesterol } \\
\text { median } \\
\text { (IQR), } \\
\text { mg/dL }\end{array}$ & $\mathbf{P}$ \\
\hline Age, years, mean (S.D.) & $48.49(16.4)$ & & & & & & & & \\
\hline Plasma lipid level, median (IQR) & & $38.1(15.1)$ & & $106.8(53)$ & & $119.5(89)$ & & $168.2(62.1)$ & \\
\hline Sex, n (\%) & & & $<0.001$ & & 0.771 & & 0.474 & & 0.760 \\
\hline Female & $46(48.4)$ & $45.2(14.6)$ & & $112.85(47.5)$ & & $126(87)$ & & $167.8(67.9)$ & \\
\hline Male & $49(51.6)$ & $34.2(13.3)$ & & $98.9(65.1)$ & & $114(83)$ & & $168.6(60)$ & \\
\hline Smoking, n (\%) & & & 0.031 & & 0.066 & & 0.619 & & 0.204 \\
\hline Never smokers & $68(71.6)$ & $40.5(16.8)$ & & $113.35(51.9)$ & & $116(87)$ & & $185.6(64.3)$ & \\
\hline Active smoker & $15(15.8)$ & $34.5(14.2)$ & & $91.7(39.3)$ & & $112(100)$ & & $149.3(61.4)$ & \\
\hline Ex-smoker & $11(11.6)$ & $30.7(14.0)$ & & $121.0(56.0)$ & & $148.0(65.0)$ & & $177.3(66.7)$ & \\
\hline \multicolumn{10}{|l|}{ Chronic diseases, $\mathrm{n}(\%)$} \\
\hline Type 2 diabetes mellitus & $29(30.5)$ & $34.95(12.7)$ & 0.011 & $111(54.8)$ & 0.599 & $151(76)$ & 0.001 & $184.6(78.7)$ & 0.357 \\
\hline Hypertension & $35(36.8)$ & $37.0(13.8)$ & 0.108 & $111(53)$ & 0.666 & $137(80)$ & 0.026 & $166(68.75)$ & 0.693 \\
\hline Coronary artery disease & $13(13.7)$ & $32.5(11.8)$ & 0.036 & $93.0(34.0)$ & 0.324 & $162(77)$ & 0.019 & $167.6(54.18)$ & 0.884 \\
\hline COPD & $8(8.4)$ & $39.55(17.8)$ & 0.850 & $106.35(65.3)$ & 0.880 & $102.0(79)$ & 0.750 & $160(92.73)$ & 0.637 \\
\hline \multicolumn{10}{|l|}{ Medications, n (\%) } \\
\hline ACE/ARB & $26(27.4)$ & $38.65(14.2)$ & 0.550 & $109(48.4)$ & 0.920 & $130.5(58)$ & 0.160 & $165.9(66.9)$ & 0.830 \\
\hline Metformin & $18(18.9)$ & $34.5(12.5)$ & 0.018 & $103.95(43.3)$ & 0.595 & $148.0(57)$ & 0.029 & $169.2(74.7)$ & 0.800 \\
\hline Statin & $8(8.4)$ & $27.3(11.0)$ & 0.006 & $89.5(25.4)$ & 0.142 & $158.0(197)$ & 0.198 & $147.6(72.4)$ & 0.232 \\
\hline Asetil salicylic acid & $13(13.4)$ & $31(22)$ & 0.382 & $95.6(38.5)$ & 0.420 & $151(92.0)$ & 0.484 & $164.8(61.9)$ & 0.549 \\
\hline Beta-blocker & $17(17.9)$ & $34.0(14.0)$ & 0.022 & $121.4(66.5)$ & 0.273 & $158(113)$ & 0.085 & $172.2(83.35)$ & 0.576 \\
\hline Calcium channel blockers & $6(6.3)$ & $32.4(10.1)$ & 0.185 & $100.45(78.7)$ & 0.387 & $96.5(153)$ & 0.862 & $164.1(108.3)$ & 0.477 \\
\hline Disease severity, n (\%) & & & 0.004 & & 0.044 & & 0.161 & & 0.091 \\
\hline Mild & $55(62.1)$ & $40.0(12.7)$ & & $106.6(46.4)$ & & $112(97)$ & & $161.7(55.7)$ & \\
\hline Moderate & $26(27.4)$ & $38.65(19.2)$ & & $130.3(59.7)$ & & $128(74)$ & & $203.2(103.3)$ & \\
\hline Severe & $10(10.5)$ & $27.1(5.9)$ & & $89.25(39.8)$ & & $148(86)$ & & $139.6(57.7)$ & \\
\hline Treatment, n (\%) & & & 0.351 & & 0.110 & $85(90)$ & 0.352 & & 0.062 \\
\hline No treatment & $5(5.3)$ & $31.3(13.4)$ & & $73.1(60.1)$ & & & & $130.5(62.1)$ & \\
\hline Hydroxychloroquine & $11(11.6)$ & $44.6(7.9)$ & & $84.5(62.5)$ & & $112(73)$ & & $152.5(69.5)$ & \\
\hline Hydroxychloroquine $+\mathrm{AZ}$ & $11(11.6)$ & $40.7(15.1)$ & & $98.1(39.0)$ & & $86(52)$ & & $151.4(51.6)$ & \\
\hline Hydroxychloroquine $+\mathrm{AZ}+\mathrm{FAV}$ & $10(10.5)$ & $30.5(22.5)$ & & $116.9(66.3)$ & & $105(61)$ & & $194.1(81.5)$ & \\
\hline Hydroxychloroquine + FAV & $4(4.2)$ & $39.2(33.8)$ & & $90.6(41.1)$ & & $137(112)$ & & $138.4(78.7)$ & \\
\hline Favipiravir (FAV) & $54(56.8)$ & $38.1(16.4)$ & & $113.3(52.3)$ & & $74.5(64)$ & & $186.5(62.85)$ & \\
\hline Corticosteroids add-on, $\mathrm{n}(\%)$ & $8(8.4)$ & $28.5(28.8)$ & 0.081 & $93(77)$ & 0.328 & $96(100)$ & 0.254 & $137.5(89.2)$ & 0.040 \\
\hline Intensive Care Unit requirement & $10(10.5)$ & $29.2(13.8)$ & 0.010 & $93.6(66.4)$ & 0.524 & $126(65)$ & 0.746 & $155.2(89.2)$ & 0.389 \\
\hline
\end{tabular}

Median HDL-C levels were significantly lower in males than females at admission (20.7 vs $45 \mathrm{mg} / \mathrm{dL}$, respectively; $\mathrm{p}<0.001)$ and were higher in never smokers than remains $(\mathrm{p}=0.031)$. Among patients who had type 2 diabetes mellitus (T2DM) and coronary artery disease (CAD) median HDL-C levels were significantly lower than those who did not have T2DM (34.95 vs $41.25 \mathrm{mg} /$ $\mathrm{dL}, \mathrm{p}=0.011)$ and CAD. $(32.55 \mathrm{vs} 39.45 \mathrm{mg} / \mathrm{dL}, \mathrm{p}=0.036)$. Median HDL-C levels were significantly lower in patients on metformin ( 34.5 vs $40.5 \mathrm{mg} / \mathrm{dL}, \mathrm{p}=0.018$ ) and betablocker ( 34.0 vs $40.5 \mathrm{mg} / \mathrm{dL}, \mathrm{p}=0.022)$ therapy than those who did not take these medications.

Median TG levels were higher in patients with T2DM (151 vs $105 \mathrm{mg} / \mathrm{dL}, \mathrm{p}=0.001$ ), hypertension (HT) (139 vs $137 \mathrm{mg} / \mathrm{dL}, \mathrm{p}=0.026)$ and CAD (162 vs $114 \mathrm{mg} / \mathrm{dL}$, $\mathrm{p}=0.019)$ than those who did not have these diseases. In addition, also patients on metformin therapy had significantly higher median TG levels than those who did not use metformin $(148 \mathrm{mg} / \mathrm{dL}$ vs $112 \mathrm{mg} / \mathrm{dL}$, $\mathrm{p}=0.029$ ).

There was no significant difference in median LDL-C and TC levels with regards to age, sex, smoking status, existing diseases and medications used.

Eight (8.4\%) of the patients were on statin treatment and no new statin therapy was initiated in any patient during hospitalization. While there was no significant difference in median LDL-C, TG, and TC levels in patients on statin treatment, HDL-C (27.3 vs $\mathrm{mg} / \mathrm{dL}$ vs $39.1, \mathrm{p}=0.006)$ was significantly lower compared to non-users.

Severe patients had significantly lower HDL-C (28.7 mg/ $\mathrm{dL}, \mathrm{p}=0.004)$ and LDL-C (93 mg/dL, $\mathrm{p}=0.044)$ levels in comparison with mild and moderate patients. The patients who require ICU during hospitalization had also lower HDL-C ( 29.2 vs $39.9 \mathrm{mg} / \mathrm{dL}, \mathrm{p}=0.010)$ than those who do not require ICU. 


\section{Plasma Lipid Values at the Time of Follow-up Visit}

The mean (SD) time period between the admission and the first visit in the COVID-19 follow-up outpatient clinic was 27.8 (12.8) days.

The median (IQR) HDL-C, LDL-C, TC and TG levels were 45.5 (15.1), 125 (43.6), 207 (67.5) and 148 (153) $\mathrm{mg} / \mathrm{dL}$, respectively. Plasma HDL-C, LDL-C, TC and TG levels on follow-up were significantly higher than those on the admission $(\mathrm{p}<0.001)$ (Figure 2).

There was no significant difference in the increment in plasma lipid levels with regards to age, sex, existing diseases and medications used (Table 2). Delta (Follow up-Admission) TG (26.9 mg/dL, p= 0.030) and delta TC $(25.5 \mathrm{mg} / \mathrm{dL}, \mathrm{p}=0.026)$ levels were significantly lower in never smokers than remains.

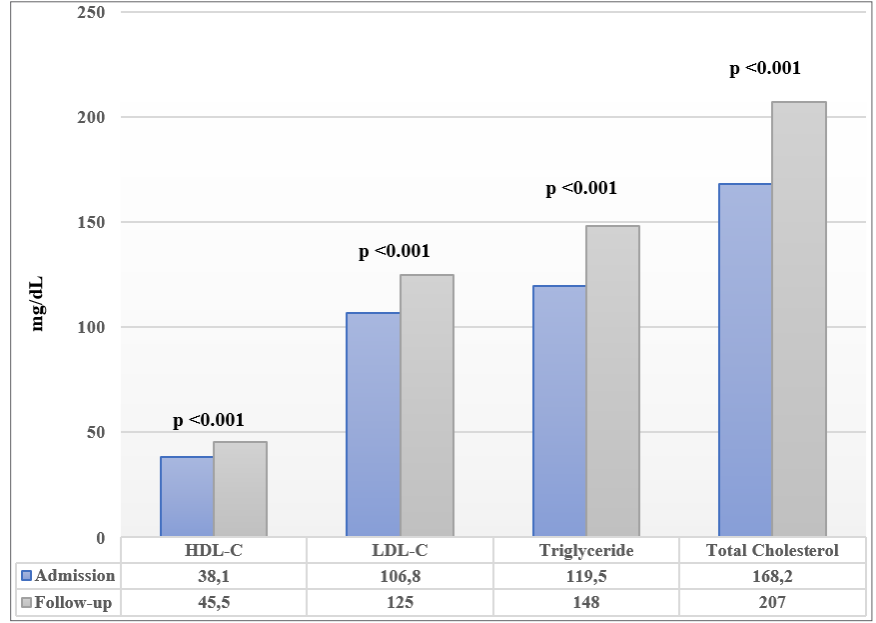

Figure 2. Plasma lipid levels of the study patients at the time of admission and in the follow-up visit

\begin{tabular}{|c|c|c|c|c|c|c|c|c|c|}
\hline & $\begin{array}{l}\text { Total, } \\
\mathbf{n}=95\end{array}$ & $\begin{array}{c}\text { Delta HDL-C } \\
\text { median } \\
\text { (IQR), } \\
\text { mg/dL }\end{array}$ & $\mathbf{P}$ & $\begin{array}{c}\text { Delta LDL-C } \\
\text { median } \\
\text { (IQR), } \\
\text { mg/dL }\end{array}$ & $\mathbf{P}$ & $\begin{array}{c}\text { Delta } \\
\text { triglycerides } \\
\text { median (IQR), } \\
\text { mg/dL }\end{array}$ & $\mathbf{P}$ & $\begin{array}{c}\text { Total } \\
\text { cholesterol } \\
\text { median (IQR), } \\
\text { mg/dL }\end{array}$ & $\mathbf{P}$ \\
\hline Age, years, mean (S.D.) & $48.4(16.4)$ & & & & & & & & \\
\hline Delta lipid levels, median (IQR) & & $5.9(9.5)$ & & $16.3(24.8)$ & & $59.3(68.2)$ & & $35.1(48.7)$ & \\
\hline Sex, $n(\%)$ & & & 0.821 & & 0.540 & & 0.203 & & 0.24 \\
\hline Female & $46(48.4)$ & $6.3(11.5)$ & & $14.8(26.5)$ & & $34.6(100.2)$ & & $28.4(40.2)$ & \\
\hline Male & $49(51.6)$ & $6.8(7.5)$ & & $18(23.2)$ & & $79.4(213.2)$ & & $40.7(54.6)$ & \\
\hline Smoking, n (\%) & & & 0.690 & & 0.372 & & 0.030 & & 0.026 \\
\hline Never smokers & $68(71.6)$ & $6.1(10.7)$ & & $14.1(26.4)$ & & $26.9(82.8)$ & & $25.5(42)$ & \\
\hline Active smokers & $15(15.8)$ & $6.6(5.4)$ & & $21.1(16.1)$ & & $134.1(343.1)$ & & $54.5(67.2)$ & \\
\hline Ex-smokers & $11(11.6)$ & $8.8(7.2)$ & & $23.4(24)$ & & $132.1(165.3)$ & & $58.7(42.9)$ & \\
\hline \multicolumn{10}{|l|}{ Chronic diseases, n (\%) } \\
\hline Type 2 diabetes mellitus & $29(30.5)$ & $6.8(6)$ & 0.913 & $12.5(24.9)$ & 0.300 & $104.5(288.3)$ & 0.285 & $38.8(64.1)$ & 0.654 \\
\hline Hypertension & $35(36.8)$ & $7.8(6.6)$ & 0.413 & $15.1(23.5)$ & 0.670 & $43.9(116.9)$ & 0.580 & $30.3(38.1)$ & 0.371 \\
\hline Coronary artery disease & $13(13.7)$ & $12(7.6)$ & 0.685 & $14.9(30.6)$ & 0.840 & $53.9(177.3)$ & 0.915 & $3.1(52.5)$ & 0.873 \\
\hline COPD & $8(8.4)$ & $6.6(9.8)$ & 0.889 & $0.2(25)$ & 0.098 & $193.7(503.8)$ & 0.029 & $42.5(106.2)$ & 0.682 \\
\hline \multicolumn{10}{|l|}{ Medications, n (\%) } \\
\hline ACE/ARB & $26(27.4)$ & $7.2(6.9)$ & 0.698 & $14.9(25.9)$ & 0.708 & $61(118.6)$ & 0.942 & $34.1(40.7)$ & 0.899 \\
\hline Metformin & $18(18.9)$ & $5.5(5.6)$ & 0.642 & $18.2(12.5)$ & 0.744 & $60.8(115.2)$ & 0.961 & $37.1(40.1)$ & 0.866 \\
\hline Statin & $8(8.4)$ & $10.4(5.4)$ & 0.274 & $27.2(33.2)$ & 0.201 & $33(164.3)$ & 0.687 & $45.5(59.9)$ & 0.562 \\
\hline Asetil salicylic acid & $13(13.4)$ & $8.7(7.7)$ & 0.443 & $22.9(28.5)$ & 0.350 & $59(133.5)$ & 0.998 & $45.2(53.7)$ & 0.490 \\
\hline Beta-blocker & $17(17.9)$ & $7.3(5.6)$ & 0.784 & $15.1(28.5)$ & 0.802 & $98.2(172)$ & 0.373 & $41.8(54.1)$ & 0.580 \\
\hline Calcium channel blockers & $6(6.3)$ & $7.8(3.1)$ & 0.470 & $20.3(17.8)$ & 0.696 & $159.8(210.6)$ & 0.136 & $60.1(45.5)$ & 0.195 \\
\hline Disease severity, n (\%) & & & 0.045 & & 0.100 & & 0.196 & & 0.112 \\
\hline Mild & $55(62.1)$ & $5.8(8.8)$ & & $14.4(21.7)$ & & $37.3(47)$ & & $27.9(37.3)$ & \\
\hline Moderate & $26(27.4)$ & $6.2(11.7)$ & & $12.6(31.4)$ & & $113.6(282.5)$ & & $41.6(68.9)$ & \\
\hline Severe & $10(10.5)$ & $14.1(13.6)$ & & $37.5(23.8)$ & & $52.5(159.5)$ & & $62.2(40.7)$ & \\
\hline Treatment, $\mathrm{n}(\%)$ & & & 0.077 & & 0.117 & & 1.710 & & 0.072 \\
\hline No treatment & & $14.7(20.6)$ & & $26.6(35.3)$ & & $78.2(190.3)$ & & $128.8(33.8)$ & \\
\hline Hydroxychloroquine & & $2.4(3.9)$ & & $2.50(18.0)$ & & $24.7(36.2)$ & & $169.7(39.1)$ & \\
\hline Hydroxychloroquine + AZ & & $5.9(3.9)$ & & $15(21)$ & & $28.5(28.5)$ & & $162.1(36.4)$ & \\
\hline Hydroxychloroquine $+\mathrm{AZ}+\mathrm{FAV}$ & & $12.3(9.1)$ & & $28.3(27)$ & & $202.7(439.2)$ & & $190.9(46.1)$ & \\
\hline Hydroxychloroquine + FAV & & $11.5(7.3)$ & & $33.8(10.9)$ & & $14.7(36.8)$ & & $154.7(45)$ & \\
\hline Favipiravir (FAV) & & $5.5(9.7)$ & & $15.2(24.9)$ & & $50.1(115.5)$ & & $189(50.2)$ & \\
\hline Corticosteroids add-on, n (\%) & $8(8.4)$ & $6.2(8.1)$ & $0.912^{*}$ & $42.4(16.9)$ & $0.004^{*}$ & $173.4(127)$ & $0.042^{*}$ & $83.3(37.2)$ & $0.004^{\star}$ \\
\hline Intensive Care Unit requirement & $10(10.5)$ & $12.1(14.2)$ & $0.242^{*}$ & $29.6(26.1)$ & $0.045^{*}$ & $191(435.7)$ & $0.341^{*}$ & $79.9(78.9)$ & $0.096^{*}$ \\
\hline
\end{tabular}


There was no statistically significant increase in lipid profiles in terms of disease severity, except HDL-C. HDL-C increased more in the severely ill patient group (delta HDL-C; 14.1, $\mathrm{p}=0.045$ ) which was lower on the admission in comparison with mild and moderate patients.

Antiviral treatment protocols for SARS-CoV-2 infection had no effects on the incremental values. Delta LDL-C (42.4 mg/dL, $\mathrm{p}=0.004)$, TG (173.4 mg/dL, $\mathrm{p}=0.042)$, and TC $(83.3 \mathrm{mg} / \mathrm{dL}, \mathrm{p}=0.004)$ levels were significantly higher in patients who have received corticosteroid therapy. Only delta LDL-C $(29.6 \mathrm{mg} / \mathrm{dL}, \mathrm{p}=0.045)$ was significantly higher in patients who require ICU during hospitalization.

Since the trend of increase in lipid levels was observed especially in patients with low lipid levels on admission, subgroups were created as above and below average lipids in the cohort, and lipid increment was reexamined according to the subgroups. In the patient group presenting with low LDL-C $(<100 \mathrm{mg} / \mathrm{dL})$ on admission, the increase in LDL was significantly higher than the rest of the cohort (Delta LDL (mean/SD); 25.5/ $22.3 \mathrm{mg} / \mathrm{dL}, \mathrm{p}=0.001)$. In patients with low TG $(<150$ $\mathrm{mg} / \mathrm{dL}$ ), a similar pattern is observed only in TG levels, but it is not statistically significant (Delta Triglyceride (mean/SD); 91.14/222.151 mg/dL, $\mathrm{p}=0.075)$. Individuals presenting with low HDL-C $(<40 \mathrm{mg} / \mathrm{dL})$ showed an increase in all parameters during the post-COVID and it was statistically significant (Delta HDL-C (mean/S.D); 9.87/8.401 mg/dL, $\mathrm{p}=<0.001$ ), (Delta LDL-C (mean/S.D); 25.49/21.06 mg/d, $\mathrm{p}=<0.001$ ), (Delta TG (mean/S.D); $86.83 / 218.27 \mathrm{mg} / \mathrm{dL}, \mathrm{p}=0.069)$, Delta TC (mean/S.D); $53.29 / 49.95 \mathrm{mg} / \mathrm{dL}, \mathrm{p}=<0.001)$.

\section{DISCUSSION}

In this real-life study, we evaluated the lipid metabolism changes in 95 hospitalized COVID-19 patients during and after the disease and the association of lipid levels with disease severity and prognosis. Alterations in lipid levels have been seen both at admission (in acute phase) and after discharge (post-COVID). While lipid levels were generally lower at admission in patients with some existing diseases (T2DM, HT, CAD), taking drugs (metformin, beta-blockers, statins) and the severity of the disease, all lipid levels were found to increase significantly after COVID.

Lipids have a valuable function in the pathophysiology of viral pneumonia. Native surfactant lipids have been recognized recently as key regulators oflung inflammation that occupy a common ground at the intersection of tissue homeostasis, host defense, and biophysics (14). It has recently been shown that diet-induced dyslipidemia alters trafficking of immune cells to the lung in a manner that may have important implications for the pathogenesis of acute lung injury, asthma, pneumonia, and other lung disorders (15). Acute inflammation caused by viruses may result in dyslipidemia in patients, and lipid metabolism is known to play an important role in the host immune response. Studies showed alterations in lipid levels in acute Epstein-Barr virus (EBV) infection (lower concentrations of apoA-I, HDL-C, TC, LDL-C), cytomegalovirus (CMV) infection (lower HDL-C) and in dengue-positive patients (lower HDL-C and LDL-C) (16-18). Remarkably, while SARS patients had lower concentrations of apoA-I in the acute phase, altered lipid metabolism has been shown in recovered SARS-CoV-1 patients even 12 years after the infection (19-20).

Detailed information on the changes in lipid profiles during COVID-19 infection is lacking. Wang et al. (21) showed that loading cells with cholesterol from blood serum using the cholesterol transport protein apolipoprotein $\mathrm{E}$ (apoE) enhances the entry of pseudotyped SARS-CoV-2 and the infectivity of the virion. Wang et al. (21) suggest that cholesterol concomitantly traffics ACE2 to viral entry sites, where SARS-CoV-2 docks in order to properly exploit entry into cells. Therefore, decreased cholesterol levels in the blood may indicate severe loading of cholesterol in peripheral tissue and escalated SARS-CoV-2 infectivity. In a recent study, abnormal lipid metabolism has been demonstrated in cured COVID-19 patients when they were about to be discharged from the hospital, indicating that viral infection and drug treatment affected the patients' systemic metabolism (11). In the related study a highresolution mass spectrometry-based lipidomic strategy was used to characterize the endogenous plasma lipids and most of the significantly changed lipids were upregulated in cured patients. A positive correlation existed between the alteration of lipids and deterioration of the disease (11). In addition, several different hypotheses related to dyslipidemia in COVID-19 have been stated such as reduction of LDL-C biosynthesis with damage of liver function caused by SARS-CoV-2, dyslipidemia due to acute inflammation (inflammatory cytokines, such as TNF-alfa, IL-6, and IL-1 beta, have been shown to modify lipid composition), increased vascular permeability, degradation of lipids due to generally elevated free radical signals in infected host cells, leakage of LDL-C into alveolar spaces to form exudates and suppress the levels of many proteins related to cholesterol metabolism $(22,23)$.

Recent studies have reported that hypolipidemia in hospitalized COVID-19 patients at the admission and the decrease in lipid levels were associated with the severity of the symptoms $(6-10,12,14,15)$. In one of the first studies 
early in the pandemic, patients with COVID-19 develop hypolipidemia as early as when they have mild symptoms and a reduction of lipid levels in patients with COVID-19 has an association with the severity of the symptoms (23).

In our study, median HDL-C was 38.1 (15.1) $\mathrm{mg} / \mathrm{dL}$ and lower in males, and lower HDL-C was correlated with the severity of COVID-19 consistent with the literature. Severe patients and the patients who required intensive care during hospitalization had lower HDL-C at the time of admission. In a study from China, HDL-C was negatively correlated with c-reactive protein (CRP) and positively correlated with lymphocytes and found and independent association with the severity of COVID-19 as a predictor (8). In another study, low concentrations of HDL-C and apoA-I at admission were significantly associated with high concentrations of CRP, prolonged hospital stay and increased disease severity (6). In an observational study, low HDL-C in COVID-19 patients was correlated with a higher risk of developing severe events (9). In a crosssectional study including 1411 hospitalized patients with COVID-19 and an available standard lipid profile prior (n:1305) or during hospitalization (n:297), patients with severe COVID-19 progression had lower HDL-C and higher TG levels before the infection (10). Median HDL-C levels were also significantly lower in patients with T2DM and CAD, which are poor prognostic factors for COVID-19 (24). On the other hand, while HDL-C and LDL-C levels upon ICU admission were low in severe COVID-19 patients, they were not found to be associated with poor outcomes (9).

In the present study, severe patients had also lower LDL-C at the admission. Lower serum levels of LDL-C and TC at admission were found as an independent predictor of LoS prolongation (2). In a small cohort of 21 patients, lipid profiles were checked before viral infections and during the course of their illness, and demonstrated that the degree of decreased LDL-C was associated with severity and mortality of the disease (25).

The most distinctive feature of this study is the follow-up lipid levels of the patients after discharge (post-COVID). All plasma lipid levels (HDL-C, LDL-C, TG and TC) on follow-up were significantly higher than those levels on the admission. The change in the pattern of increase in lipid levels was most evident in the severity of disease (HDL-C), corticosteroid use (LDL-C, TC, TG), and the need for ICU (LDL-C). Previous studies have demonstrated an increase in lipid levels during the course of the disease (in recovery phase and/or during hospitalization). In a study from China, in 68 severe cases, serum lipids were followed up three times with 5-10 days intervals during hospitalization. The median LoS was 29 days. The average levels of HDL-C, LDL-C, TG and TC, in 68 severe cases gradually and significantly increased during the following in the $2^{\text {nd }}$ (except for LDL-C) and $3^{\text {rd }}$ tests (both $\left.\mathrm{p}<0.05\right)$ (2). In another study, lipid profiles were analyzed on admission (day 1), on days 5-7 and days 15-17 after admission. From day 1 to day 15-17, TC, LDL-C, HDL-C and apoAI showed a slow upward trend in survivors, but maintained lower concentrations or showed a rapid downward trend in non-survivors (6). In a follow up study from China, LDL-C, HDL-C and TC were all significantly higher at follow-up than at the time of admission in severe/critical cases. LDL-C and TC levels were significantly higher at follow-up than at the time of admission in mild patients. The overall follow-up time was 100 days after discharge (12). In our study, HDL-C increased more in the severe patient group, although lower at presentation, confirming that HDL-C is a prognostic marker.

In our study delta (Follow up-Admission) LDL-C, TG and TC levels were significantly higher in patients who have received corticosteroid therapy. All patients were given $6 \mathrm{mg}$ /day p.o dexamethasone totally for 10 days in accordance with the local guidelines (13). Although all of the patients receiving corticosteroids were in the severely ill group, it was remarkable that only LDL-C, TG and TC increased significantly. The increase in HDL-C, which is significant in severe patients, was not significant in patients receiving corticosteroids. This situation may also support that HDL-C may be a predictor associated with the pathogenesis of COVID-19.

The clinical meaning and consequences of low lipid levels on admission (during the disease period) and the increase in the recovery period (post-COVID) are unknown. It is not known whether the surge in lipid levels consists only of a reactive process or that it may be associated with endothelial damage and vascular events during the recovery period. Whether this is a reactive increase or the effect of reverting to the patient's basal lipid profiles is unknown.

Our study has some limitations. This is a single centered study. Although the results are consistent with the literature, the sample size is small. Although the lipid levels of the patients seemed to be low at admission, it would have been better to know the basal lipid levels before the disease.

\section{CONCLUSION}

This study is valuable in that it confirms the dyslipidemia seen in previous studies in COVID-19 patients, as well as being one of the first studies in the literature in terms of showing the course of dyslipidemia in the post-COVID period. Dynamics of lipid levels before, during and after the entire disease course in a large cohort of COVID-19 patients should be monitored for better characterization of dyslipidemia. 


\section{ETHICAL DECLARATIONS}

Ethics Committee Approval: The study was carried out with the permission of Hacettepe University NonInterventional Clinical Researchs Ethics Committee (Date: 31.03.2020, Decision No: 20/353).

Informed Consent: All patients signed the free and informed consent form.

Referee Evaluation Process: Externally peer-reviewed.

Conflict of Interest Statement: The author has no conflicts of interest to declare.

Financial Disclosure: The author declared that this study has received no financial support.

Author Contributions: The author declare that they have all participated in the design, execution, and analysis of the paper, and that they have approved the final version.

Note: A part of this study has presented as e-poster on $89^{\text {th }}$ European Atherosclerosis Society (EAS) Congress in May 30 - June 2, 2021 (Abstract 1066 - Title: Can Plasma Lipid Levels Be Correlated To Severe COVID-19 Infection?)

\section{REFERENCES}

1. Feingold KR, Grunfeld C. The Effect of Inflammation and Infection on Lipids and Lipoproteins. In: Feingold KR, Anawalt B, Boyce A, Chrousos G, et al. (eds). 2019 Jan 8. Endotext [Internet]. South Dartmouth (MA): MDText.com, Inc.; 2000-

2. Qin C, Minghan H, Ziwen Z, Yukun L. Alteration of lipid profile and value of lipids in the prediction of the length of hospital stay in COVID-19 pneumonia patients. Food Sci Nutr 2020; 8: 614452.

3. Alvarez C, Ramos A. Lipids, lipoproteins, and apoproteins in serum during infection. Clin Chem 1986; 32: 142-5.

4. Filippas-Ntekouan S, Liberopoulos E, Elisaf M. Lipid testing in infectious diseases: possible role in diagnosis and prognosis. Infection 2017; 45: 575-588.

5. Grion CM, Cardoso LT, Perazolo TF, et al. Lipoproteins and CETP levels as risk factors for severe sepsis in hospitalized patients. Eur J Clin Invest 2010; 40: 330-8.

6. Li Y, Zhang Y, Lu R, et al. Lipid metabolism changes in patients with severe COVID-19. Clin Chim Acta 2021; 517: 66-73.

7. Henry BM, Szergyuk I, de Oliveira MHS, et al. Alterations in the lipid profile associate with a dysregulated inflammatory, prothrombotic, anti-fibrinolytic state and development of severe acute kidney injury in coronavirus disease 2019 (COVID-19): A study from Cincinnati, USA. Diabetes Metab Syndr 2021; 15: 863 8.

8. Hu X, Chen D, Wu L, He G, Ye W. Declined serum high density lipoprotein cholesterol is associated with the severity of COVID-19 infection. Clin Chim Acta 2020; 510: 105-10.

9. Wang G, Zhang Q, Zhao X, et al. Low high-density lipoprotein level is correlated with the severity of COVID-19 patients: an observational study. Lipids Health Dis 2020; 19: 204.

10. Masana L, Correig E, Ibarretxe D, et al; STACOV-XULA research group. Low HDL and high triglycerides predict COVID-19 severity. Sci Rep 2021; 11: 7217.

11. Bai Y, Huang W, Li Y, et al. Lipidomic alteration of plasma in cured COVID-19 patients using ultra high-performance liquid chromatography with high-resolution mass spectrometry. Biosci Rep 2021; 41: BSR20204305.
12. Li G, Du L, Cao X, et al. Follow-up study on serum cholesterol profiles and potential sequelae in recovered COVID-19 patients. BMC Infect Dis 2021; 21: 299.

13. World Health Organization. Clinical management of severe acute respiratory infection (SARI) when COVID-19 disease is suspected: interim guidance, 13 March 2020. World Health Organization. [Accessed 14 April 2020]. URL: https://apps.who. int/iris/handle/10665/331446.

14. Fessler MB, Summer RS. Surfactant lipids at the host-environment interface. metabolic sensors, suppressors, and effectors of inflammatory lung disease. Am J Respir Cell Mol Biol 2016; 54: 624-35.

15. Gowdy KM, Fessler MB. Emerging roles for cholesterol and lipoproteins in lung disease. Pulm Pharmacol Ther 2013; 26: 430 7.

16. Apostolou F, Gazi IF, Lagos K, et al. Acute infection with Epstein-Barr virus is associated with atherogenic lipid changes. Atherosclerosis 2010; 212: 607-13.

17. Fleck-Derderian S, McClellan W, Wojcicki JM. The association between cytomegalovirus infection, obesity, and metabolic syndrome in U.S. adult females. Obesity (Silver Spring) 2017; 25: 626-33.

18. Biswas HH, Gordon A, Nuñez A, Perez MA, Balmaseda A, Harris E. Lower low-density lipoprotein cholesterol levels are associated with severe dengue outcome. PLoS Negl Trop Dis 2015; 9: e0003904.

19. Wan J, Sun W, Li X, et al. Inflammation inhibitors were remarkably up-regulated in plasma of severe acute respiratory syndrome patients at progressive phase. Proteomics 2006; 6: 2886-94.

20. Wu Q, Zhou L, Sun X, et al. Altered lipid metabolism in recovered SARS patients twelve years after infection. Sci Rep 2017; 7: 9110.

21. Wang H, Yuan Z, Pavel MA, et al. Cholesterol and COVID19 lethality in elderly. bioRxiv 2020; 086249.

22. Cao X, Yin R, Albrecht H, Fan D, Tan W. Cholesterol: a new game player accelerating vasculopathy caused by SARS-CoV-2? Am J Physiol Endocrinol Metab 2020; 319: E197-E202.

23. Wei X, Zeng W, Su J, et al. Hypolipidemia is associated with the severity of COVID-19. J Clin Lipidol 2020; 14: 297-304.

24. McGurnaghan SJ, Weir A, Bishop J, et al. Risks of and risk factors for COVID-19 disease in people with diabetes: a cohort study of the total population of Scotland. Lancet Diabetes Endocrinol 2021; 9: 82-93

25.Fan J, Wang H, Ye G, et al. Letter to the Editor: Low-density lipoprotein is a potential predictor of poor prognosis in patients with coronavirus disease 2019. Metabolism 2020; 107: 154243. 